\title{
Analyzing Brain Signals by Combinatorial Optimization
}

\author{
Justin Dauwels, François Vialatte, Theophane Weber and Andrzej Cichocki
}

\begin{abstract}
We present a new method to determine the similarity (or synchrony) of a collection of multi-dimensional signals. The signals are first converted into point processes, where each event of a point process corresponds to a burst of activity of the corresponding signal in an appropriate feature space. The similarity of signals is then computed by adaptively aligning the events from the different point processes. If the point processes are similar, clusters containing one point from each time serie will naturally appear. Synchrony is then measured as a function of the size of the clusters and the distance between points within one cluster. The alignment of events is defined in a natural statistical model; the optimal clustering is obtained through maximum a posteriori inference and can be cast as a combinatorial optimization problem. As the dimension and the number of signals increase, so does the complexity of the inference task. In particular, the inference task corresponds to:
\end{abstract}

a) a dynamic program when comparing two 1-dimensional signals

b) A maximum weighted matching on a bipartite graph when comparing two $d$-dimensional signals

c) A NP-hard integer program that can be reduced to $N$ dimensional matching when comparing $N \geq 2$ signals

We show the applicability of the method by predicting the onset of Mild Cognitive Impairment (MCI) from EEG signals.

\section{INTRODUCTION}

Quantifying the interdependence between signals or time series is an important but challenging problem. Although it is straightforward to quantify linear dependencies, the extension to non-linear correlations is far from trivial. In this paper, we introduce a new exemplar-based measure of statistical interdependence between an arbitrary number of spatial point processes. It can also be applied to multidimensional signals, after they have been converted into point processes which capture "bursts" of activity of the signal in some appropriate domain. As such, we attempt to measure the synchrony of the main patterns in the data, while ignoring background activity (which can be intrinsic to the system studied, or which can be noise). This paper is organized as follows. In the following section, we outline the exemplarbased statistical model for synchrony; in Section III we describe how to perform inference in that model, and we characterize the underlying combinatorial problem. Lastly, we apply our method to detect MCI induced perturbations in EEG synchrony (Section IV). At the end of the paper, we make some concluding remarks.

J. Dauwels is supported by a fellowship from the King Baudouin Foundation and the BAEF. He is with the Laboratory for Information and Decision Systems (LIDS) at MIT (jdauwels@mit.edu). F. Vialatte and A. Cichocki are with the RIKEN Brain Science Institute (fvialatte, cia@brain.riken.jp). T. Weber is with the Operations Research Center at MIT (theo_w@mit.edu).

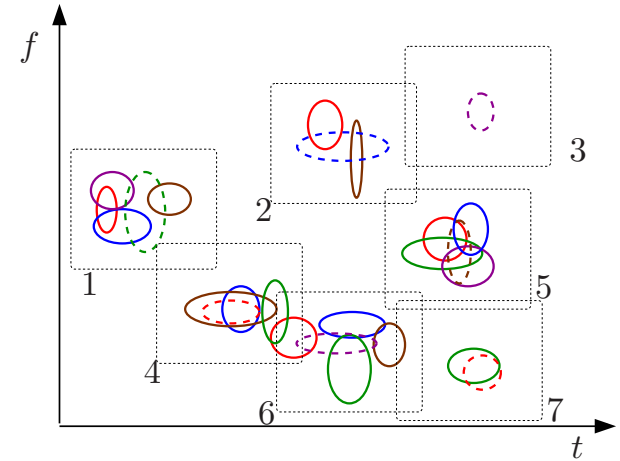

Fig. 1. Five bump models on top of each other $(N=5)$; the dashed boxes indicate clusters, the dashed ellipses correspond to exemplars.

\section{Exemplar-Based Statistical Model}

Consider $N$ signals $S_{1}, \ldots, S_{N}$ from which we extract point processes $X_{1}, \ldots, X_{N}$ by some appropriate method. Each point process $X_{i}$ is a list of $n_{i}$ points (later referred to as "events" or "bumps" of activity) in a given multidimensional set $\mathcal{S} \subseteq \mathbb{R}^{M}$, i.e., $X_{i}=\left\{X_{i, 1}, X_{i, 2}, \ldots, X_{i, n_{i}}\right\}$ with $X_{i, k} \in \mathcal{S}$ for $k=1, \ldots, n_{i}$ and $i=1 \ldots N$. As an example, consider the bump model [9] extracted from the time-frequency maps of EEG signals (see Fig. 2). The time-frequency ("wavelet") transform of each EEG signal is approximated as a sum of half-ellipsoid basis functions, referred to as "bumps" [9]; each bump is described by five parameters: time $T$, frequency $F$, width $\Delta T$, height $\Delta F$, and amplitude $W$ (as such, a bump is also a point in $\mathbb{R}^{5}$ ). We wish to quantify to which extent the $N$ resulting bump models $X_{i}=\left(\left(T_{i, 1}, F_{i, 1}, \Delta T_{i, 1}, \Delta F_{i, 1}, W_{i, 1}\right), \ldots\right.$,

$\left.\left(T_{i, n_{i}}, F_{i, n_{i}}, \Delta T_{i, n_{i}}, \Delta F_{i, n_{i}}, W_{i, n_{i}}\right)\right)$ are similar.

Intuitively speaking, $N$ signals $X_{i}$ may be considered well-synchronized if bumps appear in all models (or almost all) simultaneously, potentially with some small offset in time and frequency. In other words, if one overlays $N$ partially synchronous bump models (cf. Fig. 1 with $N=5$ ), bumps naturally appear in clusters that contain precisely one bump from all (or almost all) bump models. In the example of Fig. 2, cluster 1, 5 and 6 contain bumps from all 5 models $X_{i}$, cluster 2, 4 and 7 contains bumps from 3, 4, and 2 models respectively, and cluster 3 consists of a single bump.

This intuitive concept of similarity may readily be translated into a generative stochastic model. In that model, the $N$ point processes $X_{i}$ are treated as independent noisy observations of a hidden "mother" process $\tilde{X}$. An observed sequence $\left(X_{i}\right)_{i=1, \ldots, N}$ is obtained from $\tilde{X}$ by the following three-step procedure: 
1) COPY: generate a copy of the mother bump model $\tilde{X}$,

2) DELETION: delete some of the copied mother bumps,

3) PERTURBATION: slightly alter the position and shape of the remaining mother bump copies, amounting to the bump model $X_{i}$.

As a result, each sequence $X_{i}$ consists of "noisy" copies of a non-empty subset of mother bumps. The point processes $X_{i}$ may be considered well-synchronized if there only few deletions (cf. Step 2) and if the bumps of $X_{i}$ are "close" to the corresponding mother bumps (cf. Step 3). One way to determine the synchrony of given point processes $X_{i}$ is to first reconstruct the hidden mother process $\tilde{X}$, and to next determine the number of deletions and the average distance between the point processes $X_{i}$ and the mother process $\tilde{X}$. Inferring the mother process is a high-dimensional estimation problem, the underlying probability distribution typically has a large number of local extrema. Therefore, we will use an alternative procedure: we will assume that each cluster contains one identical copy of a mother bump, the other bumps in that cluster are noisy copies of that mother bump. The identical copy, referred to as "exemplar", plays the role of "center" or "representative" of each cluster (see Fig. 1). We will assume, without loss of generality, that there is one exemplar for each mother bump. Note that under this assumption, the mother process $\tilde{X}$ is equal to the list of all exemplars. Some point processes $X_{i}$ may additionally contain noisy copies of that mother bump, but this does not need to be the case, in other words, there might be clusters of size one, solely consisting of an exemplar (cf. cluster 3 in Fig. 1).

The exemplar-based formulation amounts to the following inference problem: given the point processes $X_{i}$, we need to identify the bumps that are exemplars and the ones that are noisy copies of some exemplar, with the constraints that an exemplar and its noisy copies all stem from different point processes. Obviously, this inference problem also has potentially many locally optimal solutions, however, in contrast to the original (continuous) inference problem, we can in practice find the global optimum by integer programming (see Section III).

We now proceed from the example of bump models to general point processes $X_{i}$, and describe the underlying stochastic model in more detail. The mother process $\tilde{X}=$ $\left\{\tilde{X}_{1}, \ldots, \tilde{X}_{M}\right\}$, which is the source of all points ("events") in $X_{1}, X_{2}, \ldots X_{N}$, is modeled as follows:

- The number $M$ of points in $\tilde{X}$ is geometrically distributed with parameter $\lambda \operatorname{vol}(S)$ :

$$
p(M)=(1-\lambda \operatorname{vol}(S))(\lambda \operatorname{vol}(S))^{M},
$$

where $\operatorname{vol}(S)$ is the multi-dimensional volume of set $S$.

- Each point $\tilde{x}_{m}$ for $m=1, \ldots, M$ is uniformly distributed in $S$ :

$$
p(\tilde{x} \mid M)=\operatorname{vol}(S)^{-M}
$$

With those two choices, the prior of the mother process $\tilde{X}$ equals:

$$
p(\tilde{x}, M)=p(M) p(\tilde{x} \mid M)=(1-\lambda \operatorname{vol}(S)) \lambda^{M} .
$$

For convenience we will in the following use the short-hand notation $p(\tilde{x})$ for $p(\tilde{x}, M)$, i.e., we will not explicitly mention the dependency on $M$.

From the mother process $\tilde{X}$, the point processes $X_{i}$ for $i=1, \ldots, N$ are generated according to the following steps:

- For each event $\tilde{X}_{m}$ in the mother process $\tilde{X}$, one of the point process $X_{i}$ with $i \in\{1, \ldots, N\}$ is chosen at random, denoted by $X_{i(m)}$, and a copy of mother event $\tilde{X}_{m}$ is created in $X_{i(m)}$; this identical copy is referred to as "exemplar". For convenience, we will adopt a uniform prior $p(i(m)=i)=1 / N$ for $i=1, \ldots, N$ (the model can be easily generalized to any prior).

Next for each event $\tilde{X}_{m}$ in the mother process $\tilde{X}$ (with $m=1, \ldots, M)$, a "noisy" copy may be created in the point processes $X_{j}$ with $j \neq i(m)$ (at most one copy per point process $X_{j}$ ). More precisely, the noisy copies are modeled as follows:

- The number $C_{m}$ of copies is modeled by a prior $p\left(c_{m} \mid \theta^{c}\right)$, parameterized by $\theta^{c}$, which in turn has a prior $p\left(\theta^{c}\right)$. In this paper, we consider as prior for $C_{m}$ : (i) a binomial distribution $\operatorname{Bi}\left(p_{s}\right)$ with $N-1$ trials and probability of success $p_{s}$; (ii) a multinomial distribution $\operatorname{Mult}(\gamma)$ with parameter $\gamma$. We adopt conjugate priors for the parameters $p_{s}$ and $\gamma$, i.e., the beta distribution $\mathrm{B}(\kappa, \lambda)$ and Dirichlet distribution $\operatorname{Di}(\zeta)$ respectively.

Note that a binomial prior $\operatorname{Bi}\left(p_{s}\right)$ for $C_{m}$ is equivalent to deleting copies of the mother events independently with probability $1-p_{s}$ (cf. DELETION step). On the other hand, if the prior $p\left(c_{m} \mid \theta^{c}\right)$ is a multinomial distribution, the copies are in general no longer deleted independently.

- Conditional on the number $C_{m}$ of copies, the copies are attributed uniformly at random to other signals $X_{j}$, with the constraints of at most one copy per signal and $j \neq$ $i(m)$; since there are $\left(\begin{array}{c}N-1 \\ c_{m}\end{array}\right)$ possible attributions $\mathcal{A}_{m} \subseteq$ $\{1, \ldots, i(m)-1, i(m)+1, \ldots, N\}$ with $\left|\mathcal{A}_{m}\right|=c_{m}$, the probability mass of an attribution $\mathcal{A}_{m}$ is $p\left(\mathcal{A}_{m} \mid c_{m}\right)=$ $\left(\begin{array}{c}N-1 \\ c_{m}\end{array}\right)^{-1}$.

- The process of generating a noisy copy $X_{i, r}$ from a mother bump $\tilde{X}_{m}$ is described by a conditional distribution $p_{x}\left(x_{i, r} \mid \tilde{x}_{m} ; \theta_{i}^{x}\right)$, parameterized by some vector $\theta_{i}^{x}$ that may differ for each point process $X_{i}$. For the sake of simplicity, the conditional distribution $p_{x}$ is assumed to be identical for all mother bumps $\tilde{X}_{m}$ and noisy copies $X_{i, r}$. The vectors $\theta_{i}^{x}$ may be treated as (mutually independent) random vectors with non-trivial priors $p\left(\theta_{i}^{x}\right)$.

In the case of bump models (cf. Fig. 1), a simple mechanism to generate copies is to slightly shift the mother bump center while the other mother bump parameters (width, height, and amplitude) are drawn from some prior distribution, independently for each copy; the latter four bump parameters could be taken 
into account in a less trivial way, but due to space constraints we omit such extensions here. The center offset may be modeled as a bivariate Gaussian random variable with mean vector $\left(\delta_{t, i}, \delta_{f, i}\right)$ and diagonal nonisotropic covariance matrix $V_{i}=\operatorname{diag}\left(s_{t, i}, s_{f, i}\right)$, and hence, $\theta_{i}^{x}=\left(\delta_{t, i}, \delta_{f, i}, s_{t, i}, s_{f, i}\right)$. For simplicity, we will assume that $s_{t, i}=s_{t}$ and $s_{f, i}=s_{f}$ for all $i$. We adopt the improper priors $p\left(\delta_{t, i}\right)=1=p\left(\delta_{f, i}\right)$ for $\delta_{t, i}$ and $\delta_{f, i}$ respectively, and conjugate priors for $s_{t}$ and $s_{f}$, i.e. scaled inverse chi-square distributions:

$$
\begin{aligned}
& p\left(s_{t}\right)=\frac{\left(s_{0, t} \nu_{t} / 2\right)^{\nu_{t} / 2}}{\Gamma\left(\nu_{t} / 2\right)} \frac{e^{-\nu_{t} s_{0, t} / 2 s_{t}}}{s_{t}^{1+\nu_{t} / 2}} \\
& p\left(s_{f}\right)=\frac{\left(s_{0, f} \nu_{f} / 2\right)^{\nu_{f} / 2}}{\Gamma\left(\nu_{f} / 2\right)} \frac{e^{-\nu_{f} s_{0, f} / 2 s_{f}}}{s_{f}^{1+\nu_{f} / 2}},
\end{aligned}
$$

where $\nu_{t}$ and $\nu_{f}$ are the degrees of freedom, and $s_{0, t}$ and $s_{0, f}$ are the width of the scaled inverse chi-square distributions, and $\Gamma(x)$ is the Gamma function. It is noteworthy that there might be a non-trivial timing and frequency offset between the bump models. The parameters $\left(\delta_{t, i}, \delta_{f, i}\right)$ are introduced in the model to account for such offsets.

For later convenience, we will introduce some more notation. The exemplar associated to mother event $\tilde{X}_{m}$ is denoted by $X_{i(m), k(m)}$, it is the event $k(m)$ in point process $X_{i(m)}$. We denote the set of pairs $(i(m), k(m))$ by $\mathcal{I}^{\text {ex }}$. A noisy copy of $\tilde{X}_{m}$ is denoted by $X_{j(m), \ell(m)}$, it is the event $\ell(m)$ in point process $X_{j(m)}$ with $j(m) \in \mathcal{A}_{m}$. We denote the set of all pairs $(j(m), \ell(m))$ associated to $\tilde{X}_{m}$ by $\mathcal{I}_{m}^{\text {copy }}$, and furthermore define $\mathcal{I}^{\text {copy }} \triangleq \mathcal{I}_{1}^{\text {copy }} \cup \cdots \cup \mathcal{I}_{M}^{\text {copy }}$ and $\mathcal{I}=\mathcal{I}^{\text {ex }} \cup \mathcal{I}^{\text {copy }}$. In this notation, the overall probabilistic model may be written as:

$$
\begin{gathered}
p(\tilde{X}, X, \mathcal{I}, \theta)=p\left(\theta^{c}\right) p\left(\theta^{x}\right)(1-\lambda \operatorname{vol}(S)) \lambda^{M} N^{-M} \\
\prod_{m=1}^{M} \delta\left(x_{i(m), k(m)}-\tilde{x}_{m}\right) p\left(c_{m} \mid \theta^{c}\right)\left(\begin{array}{c}
N-1 \\
c_{m}
\end{array}\right)^{-1} \\
\prod_{(i, j) \in \mathcal{I}_{m}^{\text {copy }}} p_{x}\left(x_{i, j} \mid \tilde{x}_{m}, \theta^{x}\right) .
\end{gathered}
$$

If the point processes $X=\left(X_{1}, \ldots, X_{N}\right)$ are wellsynchronized, almost all processes $X_{i}$ contain a copy of each mother bump $\tilde{X}_{m}$, and therefore, the sets $\mathcal{I}_{m}^{\text {copy }}$ are either of size $N-1$ or are slightly smaller. Moreover, in the case of bump models, the variances $s_{t}$ and $s_{f}$ are then small. Therefore, given point processes $X=\left(X_{1}, \ldots, X_{N}\right)$, we wish to infer $\mathcal{I}$ and $\theta$, since those variables contain information about similarity.

We gain additional insight into this inference problem by considering the logarithm of the above stochastic model:

$$
\begin{aligned}
& -\log p(\tilde{X}, X, \mathcal{I}, \theta)= \\
& -\log p\left(\theta^{c}\right)-\log p\left(\theta^{x}\right)-\log (1-\lambda \operatorname{vol}(S))-M \log \frac{\lambda}{N} \\
& -\sum_{m=1}^{M} \log \delta\left(x_{i(m), k(m)}-\tilde{x}_{m}\right)-\log \left(p\left(c_{m} \mid \theta^{c}\right)\left(\begin{array}{c}
N-1 \\
c_{m}
\end{array}\right)^{-1}\right) \\
& -\sum_{(i, j) \in \log \mathcal{I}_{m}^{\text {copy }}} \log p_{x}\left(x_{i, j} \mid \tilde{x}_{m}, \theta^{x}\right) .
\end{aligned}
$$

The term $-\log p_{x}\left(x_{i, j} \mid \tilde{x}_{m}, \theta^{x}\right)$ may be interpreted as a measure for the distance between $x_{i, j}$ and $\tilde{x}_{m}$; note that this measure is not necessarily symmetric or non-negative. If $p_{x}$ is a Gaussian distribution (as in the case of bump models), this measure is an Euclidean distance. In other applications, non-Euclidean distances may be more appropriate. The proposed algorithm can straightforwardly handle arbitrary distance measures.

Let us now consider specific choices for $p\left(c_{m} \mid \theta^{c}\right)$; if the latter is a binomial distribution with $N-1$ trials and probability of success $p_{s}$, and the prior for $p_{s}$ is a beta distribution $\mathrm{B}(\kappa, \lambda)$, we have:

$$
\begin{aligned}
& -\log p(\tilde{X}, X, \mathcal{I}, \theta)= \\
& -\log \mathrm{B}\left(p_{s} ; \kappa, \lambda\right)-\log p\left(\theta^{x}\right)-\log (1-\lambda \operatorname{vol}(S)) \\
& -M \log \frac{\lambda}{N}-\sum_{m=1}^{M} \log \delta\left(x_{i(m), k(m)}-\tilde{x}_{m}\right) \\
& -M(N-1) \log \delta-\sum_{m=1}^{M}\left(N-1-c_{m}\right) \log \frac{1-p_{s}}{p_{s}} \\
& -\sum_{(i, j) \in \mathcal{I}_{m}^{\text {copy }}} \log p_{x}\left(x_{i, j} \mid \tilde{x}_{m}, \theta^{x}\right),
\end{aligned}
$$

which can be rewritten as:

$$
\begin{aligned}
& -\log p(\tilde{X}, X, \mathcal{I}, \theta)= \\
& -\log \mathrm{B}\left(p_{s} ; \kappa, \lambda\right)-\log p\left(\theta^{x}\right)-\log (1-\lambda \operatorname{vol}(S))+\alpha M \\
& -\sum_{m=1}^{M} \log \delta\left(x_{i(m), k(m)}-\tilde{x}_{m}\right)+\beta \sum_{m=1}^{M}\left(N-1-c_{m}\right) \\
& -\sum_{(i, j) \in \mathcal{I}_{m}^{\text {copy }}} \log p_{x}\left(x_{i, j} \mid \tilde{x}_{m}, \theta^{x}\right)
\end{aligned}
$$

where

$$
\begin{aligned}
\alpha= & -\log \frac{\lambda}{N}-(N-1) \log p_{s} \\
& \text { and } \\
\beta= & \log \left(\frac{p_{s}}{1-p_{s}}\right)
\end{aligned}
$$

If $p\left(c_{m} \mid \theta^{c}\right)$ is a multinomial distribution $\operatorname{Mult}(\gamma)$ with parameter $\gamma$, and the prior for $\gamma$ is a Dirichlet distribution $\operatorname{Di}(\zeta)$, 
the expression (7) becomes:

$$
\begin{aligned}
& -\log p(\tilde{X}, X, \mathcal{I}, \theta)= \\
& -\log \operatorname{Di}(\gamma ; \zeta)-\log p\left(\theta^{x}\right)-\log (1-\lambda \operatorname{vol}(S))+\phi M \\
& -\sum_{m=1}^{M} \log \delta\left(x_{i(m), k(m)}-\tilde{x}_{m}\right)+g\left(c_{m}\right) \\
& -\sum_{(i, j) \in \log \mathcal{I}_{m}^{\text {copy }}} \log p_{x}\left(x_{i, j} \mid \tilde{x}_{m}, \theta^{x}\right) .
\end{aligned}
$$

where $\phi=-\log \frac{\lambda}{N}$, and the non-linear function $g$ is defined as:

$$
g\left(c_{m}\right)=-\log \gamma_{m}+\log \left(\begin{array}{c}
N-1 \\
c_{m}
\end{array}\right) .
$$

\section{Statistical InFERENCE}

A reasonable approach to infer $(\mathcal{I}, \theta)$ is maximum a posteriori (MAP) estimation:

$$
(\hat{\mathcal{I}}, \hat{\theta})=\underset{(\mathcal{I}, \theta)}{\operatorname{argmax}} \log p(\tilde{X}, X, \mathcal{I}, \theta) .
$$

There is no closed form expression for (13), therefore, we need to resort to numerical methods. A simple technique to try to find (13) is cyclic maximization: We first choose initial values $\hat{\theta}^{(0)}$, and then perform the following updates for $r \geq 1$ until convergence:

$$
\begin{aligned}
\hat{\mathcal{I}}^{(r)} & =\underset{\mathcal{I}}{\operatorname{argmax}} \log p\left(\tilde{X}, X, \mathcal{I}, \hat{\theta}^{(r-1)}\right) \\
\hat{\theta}^{(r)} & =\underset{\theta}{\operatorname{argmax}} \log p\left(\tilde{X}, X, \hat{\mathcal{I}}^{(r)}, \theta\right) .
\end{aligned}
$$

First we consider the update (14), which we will carry out by integer programming. Next we treat the update (15) of the parameters $\theta$.

\section{A. Integer Program}

We write the update (14) as an integer program, i.e., a discrete optimization problem with linear objective function and linear (equality and inequality) constraints. To this end, we introduce the following variables:

- $B_{i, k}$ is a binary variable equal to one iff the $k$-th event of $X_{i}$ is an exemplar.

- $B_{i, k, i^{\prime}, k^{\prime}}$ is a binary variable equal to one iff the $k$-th event of $X_{i}$ is copy of exemplar $X_{i^{\prime}, k^{\prime}}$.

- $B_{i, i^{\prime}, k^{\prime}}$ is a binary variable equal to one iff no event of $X_{i}$ is a copy of exemplar $X_{i^{\prime}, k^{\prime}}$.

Note that $b_{i, k, i, k^{\prime}}=0$ for all $k$ and $k^{\prime}$ and $b_{i, i, k^{\prime}}=1$ for all $i$ and $k^{\prime}$, since $X_{i}$ must not contain a noisy copy of a mother event $\tilde{X}_{m}$ if it already contains the exemplar associated to $\tilde{X}_{m}$.

We will first consider a binomial prior for the number of copies $C_{m}$, which directly leads to an integer program. Next we consider a multinomial prior for $C_{m}$, which results in a non-linear objective function. By introducing auxiliary variables, this objective function can be written as a linear function in the resulting augmented parameter space, and the associated combinatorial optimization problem can be formulated as an integer program, as we will briefly outline in Section III-A.2.
1) Binomial prior: We first assume that the parameters $\theta^{x}$ and $p_{s}$ of the binomial prior are constant. By substituting (9) in (14), it can be easily shown that with the above choice of variables $B$, the conditional maximization (14) may be cast as the following integer program in $B$ :

$$
\begin{aligned}
& \min _{b} C+\hat{\alpha}^{(r-1)} \sum_{i, 1 \leq k \leq n_{i}} b_{i, k}+\hat{\beta}^{(r-1)} \sum_{i, i^{\prime} \neq i, 1 \leq k^{\prime} \leq n_{i^{\prime}}} b_{i, i^{\prime}, k^{\prime}} \\
& -\sum_{i, i^{\prime}, 1 \leq k \leq n_{i}, 1 \leq k^{\prime} \leq n_{i^{\prime}}} b_{i, k, i^{\prime}, k^{\prime}} \log p_{x}\left(x_{i, k} \mid x_{i^{\prime}, k^{\prime}} ; \hat{\theta}^{(r-1)}\right)
\end{aligned}
$$

subject to

$$
\begin{array}{cl}
\forall i, k, \quad & \sum_{i^{\prime}, k^{\prime}} b_{i, k, i^{\prime}, k^{\prime}}+b_{i, k}=1 \\
\forall i, i^{\prime} \neq i, k^{\prime}, \quad & b_{i, i^{\prime}, k^{\prime}}=b_{i^{\prime}, k^{\prime}}-\sum_{1 \leq k \leq n_{i}} b_{i, k, i^{\prime}, k^{\prime}},
\end{array}
$$

where $C$ is a constant, and

$$
\begin{array}{ll}
\hat{\alpha}^{(r-1)}= & -\log \frac{\lambda}{N}-(N-1) \log \hat{p}_{s}^{(r-1)} \\
\hat{\beta}^{(r-1)}= & \log \left(\frac{\hat{p}_{s}^{(r-1)}}{1-\hat{p}_{s}^{(r-1)}}\right) .
\end{array}
$$

The sum $\sum_{i, k} b_{i, k}$ in (16) is equal to the number of exemplars $M$; therefore, the first term in (16) assigns a cost $\alpha$ to each exemplar. The second term in (16) associates a cost $\beta$ to every deletion. Indeed, if $\left(i^{\prime}, k^{\prime}\right)$ is not an exemplar, $\sum_{i} b_{i, i^{\prime}, k^{\prime}}$ is equal to zero; if $\left(i^{\prime}, k^{\prime}\right)$ is the exemplar associated to the $m$-th mother event, $\sum_{i} b_{i, i^{\prime}, k^{\prime}}=\left(N-1-c_{m}\right)$, which is the number of deletions in the $m$-th cluster. The third term assigns a cost to each copy $(i, k)$ of exemplar $\left(i^{\prime}, k^{\prime}\right)$, proportional to the "distance" $-\log p_{x}$ between both events.

The constraint (17) ensures that each event is either an exemplar or a copy of an exemplar. The constraint (18), combined with the fact that $B_{i, i^{\prime}, k^{\prime}}$ is a binary variable, encodes the following:

- $B_{i, k, i^{\prime}, k^{\prime}}$ can only be equal to one if $B_{i^{\prime}, k^{\prime}}$ is equal to one, i.e. $(i, k)$ can be a copy of $\left(i^{\prime}, k^{\prime}\right)$ iff $\left(i^{\prime}, k^{\prime}\right)$ is an exemplar,

- at most one event in $X_{i}$ can be a copy of $\left(i^{\prime}, k^{\prime}\right)$,

- $B_{i, i^{\prime}, k^{\prime}}$ is one iff $\left(i^{\prime}, k^{\prime}\right)$ is an exemplar but has no copy in $X_{i}$.

The discrete optimization problem (16)-(18) is an integer program in $B$, since the objective function (16) and constraints (17) (18) are linear in the variables $B$.

2) Multinomial prior: First we assume that the parameters $\theta^{x}$ and $\gamma$ of the multinomial prior are constant. By substituting (11) in (14), the conditional maximization (14) results 
in the following combinatorial optimization problem:

$$
\begin{aligned}
\min _{b} & \tilde{C}+\phi \sum_{i, 1 \leq k \leq n_{i}} b_{i, k} \\
& +\sum_{i^{\prime}, 1 \leq k^{\prime} \leq n_{i^{\prime}}, k^{\prime}} b_{i^{\prime}, k^{\prime}} \hat{g}^{(r-1)}\left(N-1-\sum_{i \neq i^{\prime}} b_{i, i^{\prime}, k^{\prime}}\right) \\
& -\sum_{i, i^{\prime}, 1 \leq k \leq n_{i}, 1 \leq k^{\prime} \leq n_{i^{\prime}}} b_{i, k, i^{\prime}, k^{\prime}} \log p_{x}\left(x_{i, k} \mid x_{i^{\prime}, k^{\prime}} ; \hat{\theta}^{(r-1)}\right),
\end{aligned}
$$

subject to the constraints (17) (18), where $\tilde{C}$ is an arbitrary constant and and the non-linear function $g^{(r-1)}$ is defined as:

$$
\hat{g}^{(r-1)}(c)=-\log \hat{\gamma}_{c}^{(r-1)}+\log \left(\begin{array}{c}
N-1 \\
c
\end{array}\right),
$$

for $c=0,1, \ldots, N-1$. Note that the objective function (20) is non-linear in $B$ since it involves the non-linear function $g$. We will now introduce auxiliary variables such that the objective function (20) is linear in those variables; we will then reformulate (20) as an integer program in the augmented space of variables.

Let us first point out that for an arbitrary function $f$ we can always write:

$$
f(x)=\sum_{x^{\prime} \in \mathcal{X}} f\left(x^{\prime}\right) \delta\left[x-x^{\prime}\right],
$$

with discrete (finite or infinite) set $\mathcal{X}$. By introducing variables $D_{x^{\prime}}$, we can rewrite (22) as:

$$
f(x)=\sum_{x^{\prime} \in \mathcal{X}} f\left(x^{\prime}\right) d_{x^{\prime}},
$$

with the constraint $d_{x^{\prime}}=\delta\left[x-x^{\prime}\right]$. The key observation here is that (23) is linear in $D_{x^{\prime}}$.

In this vein, we introduce the binary variables $D_{v, i^{\prime}, k^{\prime}}$ and rewrite the objective function (20) as:

$$
\begin{aligned}
\min _{b} \phi & \sum_{i, 1 \leq k \leq n_{i}} b_{i, k}+\sum_{v, i^{\prime}, 1 \leq k^{\prime} \leq n_{i^{\prime}}} g_{v}^{(r-1)} d_{v, i^{\prime}, k^{\prime}} \\
& -\sum_{\substack{i, i^{\prime}, 1 \leq k \leq n_{i}, 1 \leq k^{\prime} \leq n_{i^{\prime}} \\
b_{i, k, i^{\prime}, k^{\prime}}}} \log p_{x}\left(x_{i, k} \mid x_{i^{\prime}, k^{\prime}} ; \theta\right)+\tilde{C},
\end{aligned}
$$

where $g_{v}^{(r-1)}=g^{(r-1)}(N-1-v)$. This alternative formulation is equivalent to the original expression (20) iff $D_{v, i^{\prime}, k^{\prime}}$ equals one if both $v=\sum_{i \neq i^{\prime}} b_{i, i^{\prime}, k^{\prime}}$ and $b_{i^{\prime}, k^{\prime}}=1$, and is zero otherwise. We express those constraints on $D_{v, i^{\prime}, k^{\prime}}$ as follows:

$$
\begin{aligned}
v-\sum_{i \neq i^{\prime}} b_{i, i^{\prime}, k^{\prime}} & \leq a_{v, i^{\prime}, k^{\prime}}, \\
\sum_{i \neq i^{\prime}} b_{i, i^{\prime}, k^{\prime}}-v & \leq a_{v, i^{\prime}, k^{\prime}}, \\
\sum_{v} d_{v, i^{\prime}, k^{\prime}} & \leq N\left(1-d_{v, i^{\prime}, k^{\prime}, k^{\prime}}=b_{i^{\prime}, k^{\prime}},\right.
\end{aligned}
$$

where $A_{v, i^{\prime}, k^{\prime}}$ are additional auxiliary binary variables. The first two constraints encode that $a_{v, i^{\prime}, k^{\prime}} \geq\left|v-\sum_{i \neq i^{\prime}} b_{i, i^{\prime}, k^{\prime}}\right|$.
If $v \neq \sum_{i \neq i^{\prime}} b_{i, i^{\prime}, k^{\prime}}$, the variable $A_{v, i^{\prime}, k^{\prime}}$ is strictly positive, and from the third inequality it follows that $D_{v, i^{\prime}, k^{\prime}}$ equals zero. On the other hand, if $v=\sum_{i \neq i^{\prime}} b_{i, i^{\prime}, k^{\prime}}$, the first two constraints no longer force $A_{v, i^{\prime}, k^{\prime}}$ to be non-zero, and they do not impose any constraint on $D_{v, i^{\prime}, k^{\prime}}$. However, from the fourth constraint it follows that if $b_{i^{\prime}, k^{\prime}}=1$ and hence if $\left(i^{\prime}, k^{\prime}\right)$ is an exemplar, one of the $D_{v, i^{\prime}, k^{\prime}}$ (with fixed $i^{\prime}$ and $k^{\prime}$ ) is equal to one. By setting $D_{v, i^{\prime}, k^{\prime}}$ equal to one if $v=\sum_{i \neq i^{\prime}} b_{i, i^{\prime}, k^{\prime}}$ and zero otherwise, one fulfills then all four constraints. If $b_{i^{\prime}, k^{\prime}}=0$ and hence if $\left(i^{\prime}, k^{\prime}\right)$ is not an exemplar, all $D_{v, i^{\prime}, k^{\prime}}$ (with fixed $i^{\prime}$ and $k^{\prime}$ ) are equal to zero. By setting all $D_{v, i^{\prime}, k^{\prime}}$ equal to zero, one in that case fulfills all four constraints.

In summary: the non-linear combinatorial optimization problem with objective (20) and constraints (17) (18) is equivalent to the integer program with objective (24) and constraints (17) (18) combined with (25)-(28).

3) Complexity: In the case $N=2$, it can be seen that the combinatorial optimization problem (14) can be reduced to a bipartite maximum weighted matching optimization problem, which can be solved in polynomial time through several methods: linear programming relaxation, EdmondKarp algorithm, or max-product message-passing algorithm detailed in [5]. For $N>2$, (14) is very similar to solving a maximum weighted $N$-dimensional matching. For the purpose of understanding the combinatorial hardness of the problem, we show that for $N \geq 5$, the maximum 3-dimensional matching problem can be reduced to (14) when forgoing the euclidean costs assumptions. Since maximum 3-dimensional matching is NP-hard, it results that (14) (with general costs) is also NP-hard. The problem is actually NP-hard for $N \geq 3$, but the proof is more involved and beyond the scope of this paper. Therefore, the extension from 2 time series to more than 2 is far from trivial. Nevertheless, as we will discuss later, we were able to solve the problem (14) for our purpose in reasonable time using integer programming techniques.

Proposition 1: The combinatorial problem (15) is NPhard if $N \geq 5$.

We include a sketch of the proof ; it is based on a reduction from maximum weighted 3-dimensional matching optimization, which is known to be NP-hard and APX-hard [20] [21].

Proof: Let $T \subset X \times Y \times Z$, where $X, Y, Z$ are disjoint sets. Consider the following "time-series" $X^{\prime}, Y^{\prime}, Z^{\prime}, T^{\prime}, U^{\prime}$. For every $x \in X$ (resp. $y \in Y, z \in Z$ ), create two corresponding bumps $x \in X^{\prime}$ and $\tilde{x} \in U^{\prime}$ (resp. $y \in Y^{\prime}$, $\left.z \in Z^{\prime}, \tilde{y} \in U, \tilde{z} \in U^{\prime}\right)$ and for every $t=(x, y, z) \in T$, create two bumps $t \in T^{\prime}$ and $\tilde{t} \in U^{\prime}$. Set the cost function as follows:

- $p_{s}=1-\epsilon$, where $\epsilon$ is an extremely small positive constant (practically 0)

- $\lambda=N \exp (1)$

- For any $t=(x, y, z) \in T$, let $s_{x, t}=s_{y, t}=s_{z, t}=0$. For any bump $b \in X^{\prime} \cup Y^{\prime} \cup Z^{\prime} \cup T^{\prime}$, let $s_{b, \tilde{b}}=\beta$. For any other two bumps $b_{1}, b_{2}$, let $s_{b_{1}, b_{2}}$ be equal to $M$, where $\mathrm{M}$ is a very large positive constant (pratically, $+\infty)$. 
The first two assumptions effectively set $\alpha$ to -1 and $\beta$ to a very large number. Note the following: all bumps in $U^{\prime}$ have to be exemplars (because for any bump $u \in U^{\prime}$ and any other bump $b, s_{u, b}$ is infinite). The total cost of bumps in $U^{\prime}$ being exemplars is therefore an additive constant which does not change the solution. Moreover, for any bump $u \in U^{\prime}$, there exists a unique bump $b \in X^{\prime} \cup Y^{\prime} \cup Z^{\prime} \cup T^{\prime}$ that can be assigned to it (i.e. for any $b^{\prime} \neq b, s_{b^{\prime}, u}=+\infty$ ). If this bump $b$ is assigned $u$, the assignment cost $s_{b, u}$ is $\beta$, and since 4 bumps are missing in the cluster, the cost of missing bumps is $4 \beta$. The total cluster cost would therefore be $5 \beta$. If the bump $b$ is not assigned to $u, 5$ bumps are missing in the cluster and the total cluster cost is again $5 \beta$. Therefore, bumps $b \in X^{\prime} \cup Y^{\prime} \cup Z^{\prime} \cup T^{\prime}$ can be assigned to their corresponding exemplar in $U^{\prime}$ without changing the total cost. For this reason, exemplars in $U^{\prime}$ can be considered as "fake exemplars" (they serve as bins for unmatched bumps in $X^{\prime}, Y^{\prime}, Z^{\prime}$ and $T^{\prime}$ ). The next step consists in observing all other exemplars have to be in $T^{\prime}$. Indeed, for any bump $b_{1} \in X^{\prime} \cup Y^{\prime} \cup Z^{\prime}$, and any other bump $b_{2}, s_{b_{2}, b_{1}}$ is infinite. Moreover, since $\alpha=-1$, the optimization effectively aims at maximizing the number of exemplars in $T^{\prime}$. Since the cost of missing bumps $\beta$ is very large, all "real" clusters (with exemplars in $T^{\prime}$ ) have to contain a bump from each time serie $X^{\prime}, Y^{\prime}, Z^{\prime}$. Let $t=(x, y, z) \in T$. Then the only possible cluster for exemplar $t \in T^{\prime}$ consists of the corresponding bumps $x, y, z$ in $X^{\prime}, Y^{\prime}, Z^{\prime}$ (all other assignements bring the cost up to infinity). Finally, since each bump in $X^{\prime}, Y^{\prime}, Z^{\prime}$ can only be assigned to one exemplar in $T^{\prime}$, the clusters differ in each coordinate. It finally follows that the set of real clusters is the maximum 3-dimensional matching of $T \subset$ $X \times Y \times Z$.

In practice (see the applications of Section IV), we were often able to solve the corresponding integer program very efficiently: for integer programs with more than 10'000 variables and 5'000 constraints, the solution of a given was obtained in less than 1 second on a fast processor $(3 \mathrm{GHz})$. The total running time of the algorithm (iterations of equations (14) and (15) until convergence) was under 7 seconds on average. This is perhaps surprising, especially in the light of the fact that message-passing algorithms did relatively poorly on the problem (slow convergence, relatively weak solutions). We believe that the good performance of the IP stems from the relatively good performance of the LP relaxation. Typically, in our instances, the LP relaxation approximated the optimal cost within $3 \%$; and a third of the positive components of the optimal LP solution were integer.

\section{B. Parameter Estimation}

We now consider the update (15), i.e., estimation of the parameters $\theta=\left(\theta^{x}, \theta^{c}\right)$. The estimate $\hat{\theta}^{(r+1)}=$ $\left(\hat{\theta}^{x^{(r+1)}}, \hat{\theta}^{c^{(r+1)}}\right)(15)$ is often available in closed-form. This is in particular the case for the parametrization $\theta_{i}^{x}=$ $\left(\delta_{t, i}, \delta_{f, i}, s_{t}, s_{f}\right)$. The point estimates $\hat{\delta}_{t, i}^{(r+1)}$ and $\hat{\delta}_{f, i}^{(r+1)}$ are the (sample) mean of the timing and frequency offset respectively, computed between all noisy copies in $X_{i}$ and their associated exemplars:

$$
\begin{aligned}
& \hat{\delta}_{t, i}^{(r)}=\frac{1}{n_{i}^{(r)}} \sum_{k, i^{\prime}, k^{\prime}} \hat{b}_{i, k, i^{\prime}, k^{\prime}}^{(r)}\left(T_{i, k}-T_{i^{\prime}, k^{\prime}}\right) \\
& \hat{\delta}_{f, i}^{(r)}=\frac{1}{n_{i}^{(r)}} \sum_{k, i^{\prime}, k^{\prime}} \hat{b}_{i, k, i^{\prime}, k^{\prime}}^{(r)}\left(F_{i, k}-F_{i^{\prime}, k^{\prime}}\right),
\end{aligned}
$$

where $n_{i}^{(r)}$ is the number of noisy copies in $X_{i}$ :

$$
n_{i}^{(r)}=\sum_{k, i^{\prime}, k^{\prime}} \hat{b}_{i, k, i^{\prime}, k^{\prime}}^{(r)}=n_{i}-\sum_{k} \hat{b}_{i, k}^{(r)} .
$$

The estimates $\hat{s}_{t}^{(r)}$ and $\hat{s}_{f}^{(r)}$ are obtained as:

$$
\begin{aligned}
& \hat{s}_{t}^{(r)}=\frac{\nu_{t} s_{0, t}+n^{(r)} \hat{s}_{t, \text { sample }}^{(r)}}{\nu_{t}+n^{(r)}+2} \\
& \hat{s}_{f}^{(r)}=\frac{\nu_{f} s_{0, f}+n^{(r)} \hat{s}_{f, \text { sample }}^{(r)}}{\nu_{f}+n^{(r)}+2},
\end{aligned}
$$

where $s_{t \text {,sample }}^{(r)}$ and $s_{f \text {,sample }}^{(r)}$ are computed over all exemplars and their noisy copies:

$$
\begin{aligned}
& \hat{s}_{t, \text { sample }}^{(r)}=\frac{1}{n^{(r)}} \sum_{i, k, i^{\prime}, k^{\prime}} \hat{b}_{i, k, i^{\prime}, k^{\prime}}^{(r)}\left(T_{i, k}-T_{i^{\prime}, k^{\prime}}\right)^{2}, \\
& \hat{s}_{f, \text { sample }}^{(r)}=\frac{1}{n^{(r)}} \sum_{i, k, i^{\prime}, k^{\prime}} \hat{b}_{i, k, i^{\prime}, k^{\prime}}^{(r)}\left(F_{i, k}-F_{i^{\prime}, k^{\prime}}\right)^{2},
\end{aligned}
$$

and $n^{(r)}$ is the total number of noisy copies:

$n^{(r)}=\sum_{i, k, i^{\prime}, k^{\prime}} \hat{b}_{i, k, i^{\prime}, k^{\prime}}^{(r)}=\sum_{i} n_{i}-\sum_{i, k} \hat{b}_{i, k}^{(r)}=\sum_{i} n_{i}-\hat{M}^{(r)}$.

The parameter $p_{s}$ of the binomial prior for the number of copies $C_{m}$ is estimated as:

$$
\hat{p}_{s}^{(r)}=\frac{\kappa+\sum_{i} n_{i}-\hat{M}^{(r)}-1}{\kappa+\lambda+\hat{M}^{(r)}-2} .
$$

The parameter $\gamma$ of the multinomial prior for number of copies $C_{m}$ is estimated as:

$$
\hat{\gamma}_{j}^{(r)}=\frac{\zeta_{i}-1+\sum_{i^{\prime}, k^{\prime}} \hat{b}_{i^{\prime}, k^{\prime}}^{(r)} \delta\left[\sum_{i, k} \hat{b}_{i, k, i^{\prime}, k^{\prime}}^{(r)}-j\right]}{\sum_{i} \zeta_{i}-N+\sum_{i, k} \hat{b}_{i, k}^{(r)}},
$$

for $j=0,1, \ldots, N-1$.

\section{Diagnosis of MCI FROM EEG}

For the sake of comparison, we used the same EEG data as in [5], i.e., rest eyes-closed EEG data recorded from 21 sites on the scalp based on the 10-20 system [18] with sampling frequency of $200 \mathrm{~Hz}$ and band-pass filter between 4 and $30 \mathrm{~Hz}$. The subjects consist of two groups: (i) 22 patients suffering from mild cognitive impairment (MCI), who subsequently developed mild AD; (ii) a control set of 38 age-matched, healthy subjects who had no memory or other cognitive impairments. Pre-selection was conducted to ensure that the data were of a high quality, as determined by the presence of at least 20 s of artifact free data. We aggregated the 21 bump models in five regions (frontal, 


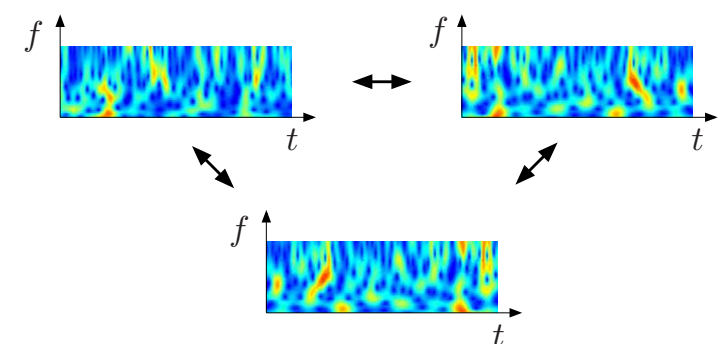

(a) Time-frequency maps.

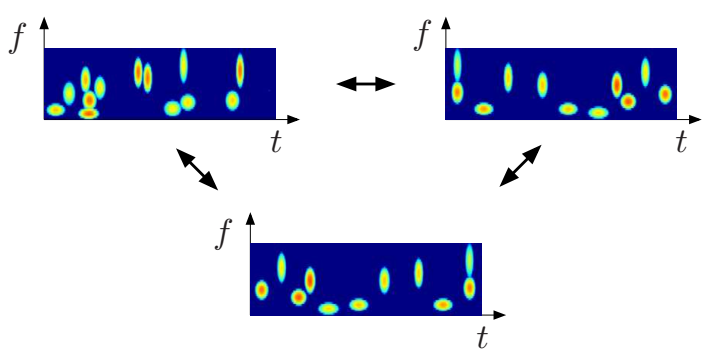

(b) Bump models.

Fig. 2. Similarity of three EEG signals $(N=3)$; from their time-frequency transforms (top), one extracts two-dimensional point processes ("bump models"; bottom), which are then aligned by the proposed algorithm.

temporal left and right, central, occipital) by means of the aggregation algorithm described in [9], resulting in a bump model for each of those five regions $(N=5)$.

A large variety of classical synchrony measures (more than 30 in total) have in [5] been applied to both data sets with the aim of detecting MCI induced perturbations in EEG synchrony; none of those classical measures except full frequency Directed Transfer Function (ffDTF) [14], which is a Granger causality measure, was able to detect significant loss of EEG synchrony in MCI patients. More precisely, all measures amount to Mann-Whitney $p$-values larger than 0.005 with the exception of ffDTF $(p=0.0012)$. On the other hand, the stochastic-event-synchrony measure $\rho$, proposed in [5], resulted in significant differences between both subject groups $\left(p=2.1 \cdot 10^{-4}\right)$. This seems to indicate that there is an increase of unsynchronized activity in MCI patients.

The results from our exemplar-based approach are summarized in Table I; the results are for the binomial prior, the results for the multinomial prior closely agree. In other words, the choice of prior does again not seem to be crucial. We adopted constant parameters, because time-varying parameters are less suitable since we consider spontaneous EEG. We studied the following statistics:

- Posterior distribution $p\left(c_{m}=i \mid X\right)=p_{i}^{c}$ of the number of copies of each exemplar $c_{m}$, parameterized by $\left(p_{0}^{c}, p_{1}^{c}, \ldots, p_{4}^{c}\right)$,

- $\bar{c}_{m}$ : average number of copies per cluster,

- $s_{t}$ : variance in time domain ("time jitter"),

- $s_{f}$ : variance in frequency domain ("frequency jitter"),

- $\Delta \bar{T}$ : average width of bumps,

- $\Delta \bar{F}$ : average height of bumps,

- $\bar{F}$ : average frequency of bumps.

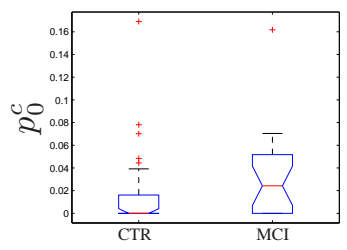

(a) $p_{0}^{c}$

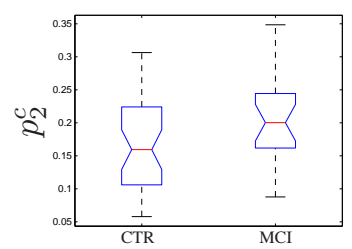

(c) $p_{2}^{c}$

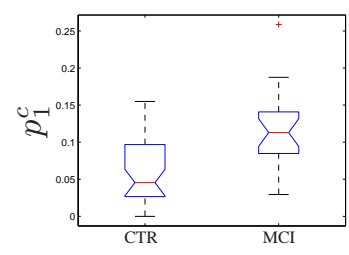

(b) $p_{1}^{c}$

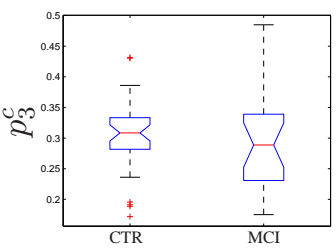

(d) $p_{3}^{c}$

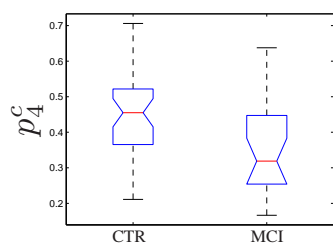

(e) $p_{4}^{c}$

Fig. 3. Box plots for posterior distribution $p\left(c_{m}=i \mid X\right)=p_{i}^{c}$.

We also consider the linear combination $h^{c}$ of all parameters $p_{i}^{c}$ that optimally separates both subject groups. Interestingly, the latter statistic amounts to about the same $p$-value as the index $\rho$ of SES [5]. The posterior $p\left(c_{m} \mid X\right)$ mostly differs in $p_{1}^{c}, p_{2}^{c}$ and $p_{4}^{c}$ (see Fig. 3): in MCI patients, the number of clusters of size five $\left(p_{4}^{c}\right)$ significantly decreases; on the other hand, the number of clusters of size one $\left(p_{1}^{c}\right)$ and two $\left(p_{2}^{c}\right)$ significantly increases. This explains and confirms the observed increase of $\rho$ in MCI patients [5]. Combining $h^{c}$ with ffDTF and $\Delta \bar{T}$ (or $\bar{c}_{m}$ with ffDTF and $\Delta \bar{T}$ ) allows to separate the two groups quite well (more than $90 \%$ correctly classified), as shown in Fig. 5; this is far better than what can be achieved by means of classical similarity measures (about $75 \%$ correctly classified). Classification rates between 80 and $85 \%$ can be obtained by combining two features (see Fig. 4(a)).

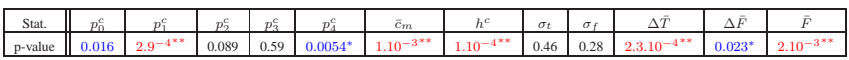

\section{TABLE I}

SENSITIVITY OF MULTIVARIATE SES FOR DIAGNOSING MCI (P-VALUES FOR MANN-WHITNEY TEST; * AND ** INDICATE $p<0.05$ AND $p<0.005$ RESPECTIVELY).

\section{CONClusion}

We proposed an approach to determine the similarity of multiple (one- and multi-dimensional) point processes; it is based on an exemplar-based statistical model that describes 


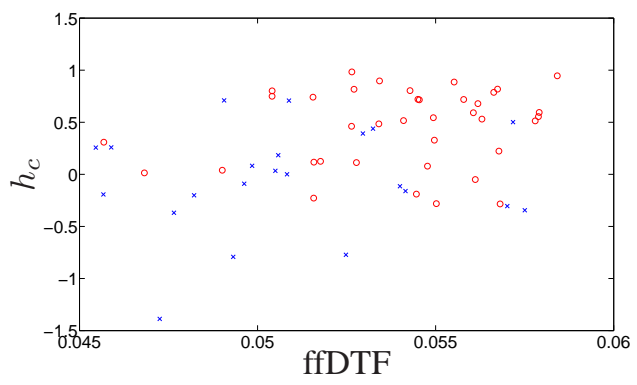

(a) $h_{c}$ vs. ffDTF

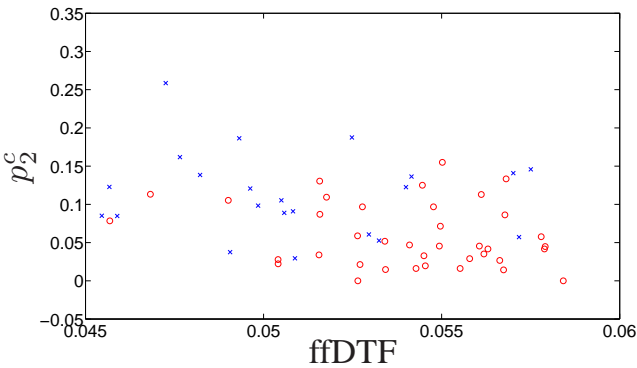

(b) $p_{2}^{c}$ vs. ffDTF

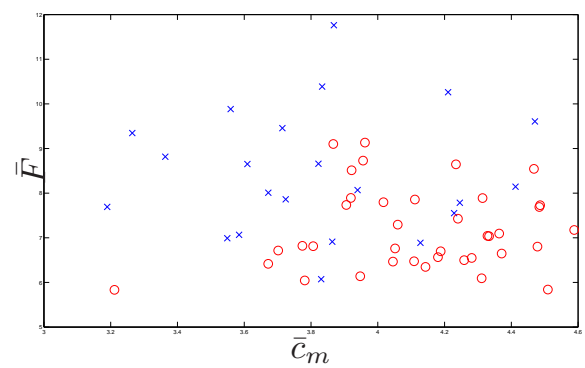

(c) $\bar{F}$ vs. $\bar{c}_{m}$

Fig. 4. Combination of two features.

how the point processes are related through a common hidden "mother" process. The similarity of the point processes is determined by performing inference in that model by means of integer programming techniques in conjunction with point estimation of the parameters. The proposed technique may be used for various applications in neuroscience (e.g., in brain-computer interfaces, analysis of spike data), biomedical signal processing, and beyond.

\section{REFERENCES}

[1] E. Pereda, R. Q. Quiroga, and J. Bhattacharya, "Nonlinear multivariate analysis of neurophsyiological signals," Progress in Neurobiology, 77 (2005) 1-37.

[2] G. Buzsáki, Rhythms of the Brain, Oxford University Press, 2006.

[3] T. Womelsdorf, J.M. Schoffelen, R. Oostenveld, W. Singer, R. Desimone, A.K. Engel, P. Fries, "Modulation of neuronal interactions through neuronal synchronization," Science, 316:1609-1612.

[4] P. Uhlhaas and W. Singer, "Neural synchrony in brain disorders: relevance for cognitive dysfunctions and pathophysiology," Neuron, 52:155-168, 2006.

[5] J. Dauwels, F. Vialatte, T. Rutkowski, and A. Cichocki, "Measuring neural synchrony by message passing," Advances in Neural Information Processing Systems 20, MIT Press.

[6] B. Frey and D. Dueck, "Clustering by passing messages between data points," Science, vol. 315, No. 5814, pp. 972-976, 2007.

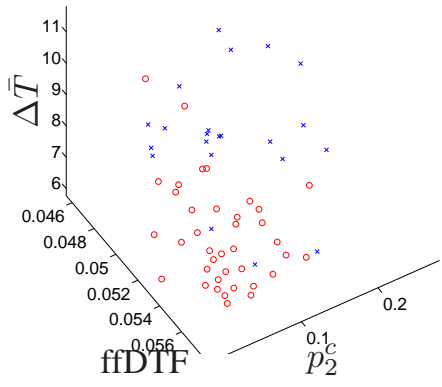

(a) $\Delta \bar{T}$ vs. ffDTF vs. $p_{2}^{c}$

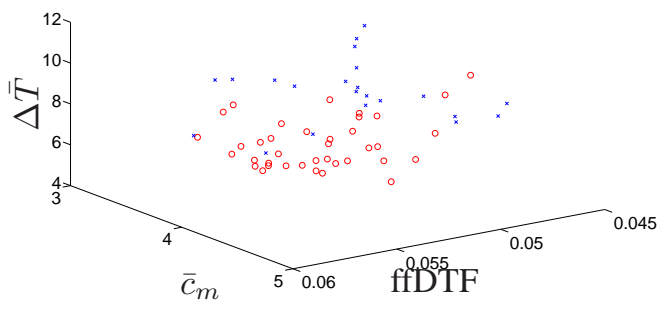

(b) $\Delta \bar{T}$ vs. $\bar{c}_{m}$ vs. ffDTF

Fig. 5. Combination of three features.

[7] B. Frey and D. Dueck, "Mixture modelling by affinity propagation," In Advances in Neural Information Processing Systems 18, MIT Press.

[8] D. Lashkari and P. Golland, "Convex clustering with exemplar-based models," In Advances in Neural Information Processing Systems 20, MIT Press.

[9] F. Vialatte, C. Martin, R. Dubois, J. Haddad, B. Quenet, R. Gervais, and G. Dreyfus, "A Machine learning approach to the analysis of time-frequency maps, and its application to neural dynamics," Neural Networks, 2007, 20:194-209.

[10] J. Dauwels, F. Vialatte, and A. Cichocki, "A comparative study of synchrony measures for the early detection of AD," Proc. ICONIP 2007, Kitakyushu, September 2007.

[11] J.-P. Lachaux, E. Rodriguez, J. Martinerie, and F. J. Varela, "Measuring Phase Synchrony in Brain Signals," Human Brain Mapping 8:194208 (1999).

[12] A. Kraskov, H. Stögbauer, and P. Grassberger, "Estimating Mutual Information," Phys. Rev. E 69 (6) 066138, 2004.

[13] S. Aviyente, "A Measure of Mutual Information on the TimeFrequency Plane," Proc. of ICASSP 2005, vol. 4, pp. 481-484, March 18-23, 2005, Philadelphia, PA, USA.

[14] M. Kamiński and Hualou Liang, "Causal Influence: Advances in Neurosignal Analysis," Critical Review in Biomedical Engineering, 33(4):347-430 (2005).

[15] I. J. Schoenberg, "Spline functions and the problem of graduation," Mathematics 52: 974-50.

[16] D. MacKay, Information Theory, Inference, and Learning Algorithms, Cambridge University Press, 2003.

[17] H.-A. Loeliger, "An introduction to factor graphs," IEEE Signal Processing Magazine, Jan. 2004, pp. 28-41.

[18] P. Nunez and R. Srinivasan, Electric Fields of the Brain: The Neurophysics of EEG, Oxford University Press, 2006.

[19] http://www.ilog.com/products/optimization/

[20] V. Kann, "Maximum bounded 3-dimensional matching is MAX SNPcomplete", Inform. Process. Lett. 37, 27-35, 1991.

[21] M.R. Garey and D.S. Johnson, Computers and Intractability: A Guide to the Theory of NP-Completeness, W. H. Freeman \& Co., 1979. 